\title{
A video system for investigating breathing disorders during sleep
}

\author{
C J Griffiths, B G Cooper, G J Gibson
}

\begin{abstract}
A system has been developed for investigating breathing during sleep that superimposes physiological signals on a video image of the patient, with the combined image plus sound recorded on video tape for later analysis. Signals normally displayed include oxygen saturation, airflow, chest wall motion, electroencephalogram, and electrooculogram; but others can be recorded if desired. The information is displayed on a timebase appropriate for the recognition and analysis of respiratory events during sleep. In addition, use is made of normally invisible video lines to record the analogue voltage waveforms so that on replay this information can be displayed on a fast timebase for analysing the more rapidly chainging waveforms of electrophysiological signals. The system allows detailed polysomnography to be performed in the normal ward setting with the subject monitored overnight by the nursing stafi. Subsequent analysis of the synchronised video, audio, and analogue signals allows measurement of the conventional indices obtained by polysomnography and aids their interpretation.
\end{abstract}

The recognition of sleep apnoea as a major clinical problem has necessitated the development of various methods for investigating breathing problems during sleep. Although simple "screening" investigations, such as the measurement of nocturnal oxygen saturation $\left(\mathrm{SaO}_{2}\right)$ alone, ${ }^{12}$ are useful, their role is necessarily limited and most patients in whom sleep apnoea is suspected on clinical grounds require fuller investigation. Detailed polysomnography, as practised in most centres, includes the recording of multiple signals relating to oxygenation, airflow, and chest wall motion and of electrophysiological data, with the information recorded on a chart recorder or on magnetic tape or directly by a computer. Usually the subject is under continuous supervision and "there is a consensus among clinical polysomnographers that ... the patient needs to be monitored throughout the night by a skilled laboratory technician."3 Such investigations are therefore highly labour intensive. In view of the rapid increase in referrals noted by all centres investigating disorders of breathing during sleep, there is a need for a less costly system that allows all the relevant data to be obtained without necessarily requiring the presence of a technician throughout the night. We have developed a video system that offers these advantages.

\section{Methods and results}

In essence, the system (fig 1) allows the superimposition of the physiological data as coloured waveforms on a monochrome video image of the sleeping patient; the combined image, with sound, is recorded on video tape for later analysis.

The superimposition of waveforms is achieved with an "analogue and video mixer" device, which was developed originally for urodynamic studies, where bladder pressure and flow data are superimposed on a video fluoroscopic image of the bladder. ${ }^{4}$ The technical principles of this device have been described. ${ }^{5} \mathrm{Up}$ to eight channels of data can be superimposed, each with a pixel resolution of 256 (vertical) by 512 (horizontal). For sleep studies a sample rate of about $6 \mathrm{~Hz}$ is used, giving a display of 80 seconds of data, the most recent at the right of the screen and scrolling to the left. This timebase was chosen as convenient for the display of respiratory information, including episodes of apnoea, hypopnoea, and oxygen desaturation. Such a timebase is, however, inappropriate for detailed inspection of the faster changing waveforms of electrophysiological signals.

To overcome this problem (and also to allow the selective production of hard copy), the physiological signals are recorded on the video tape with a newly developed "analogue to video to analogue" (AVA) encoder-decoder device. The basic principle is illustrated in figure 2. Video lines that do not contribute to the image are used to carry the analogue data in an encoded manner (these are the lines which carry Teletext information in broadcast television). Sixteen lines are available; normally we use eight, the other eight being available for additional signals if required. One line is used per signal, the length of the "illuminated" part of the line being varied in proportion to the voltage of the signal at that instant. The set of lines is scanned $\mathbf{5 0}$ times a second and this determines the sample rate for each channel of data. The maximum signal
Freeman Hospital, Newcastle upon Tyne Reprint requests to: Mr Grifiths Accepted 20 September 1990 
Figure 1 Block diagram showing the signals recorded and the main components of the video sleep study system (see text).

Figure 2 Schematic diagram of the basic principles of the analogue to video to analogue encoder-decoder device, which records the analogue signals on to the video tape using lines not appearing in the normal image. Each of eight signals uses one line; the illuminated length of each line (indicated by thickened line in the figure) is proportional to the voltage of the signal, which is sampled at a frequency of $50 \mathrm{~Hz}$.

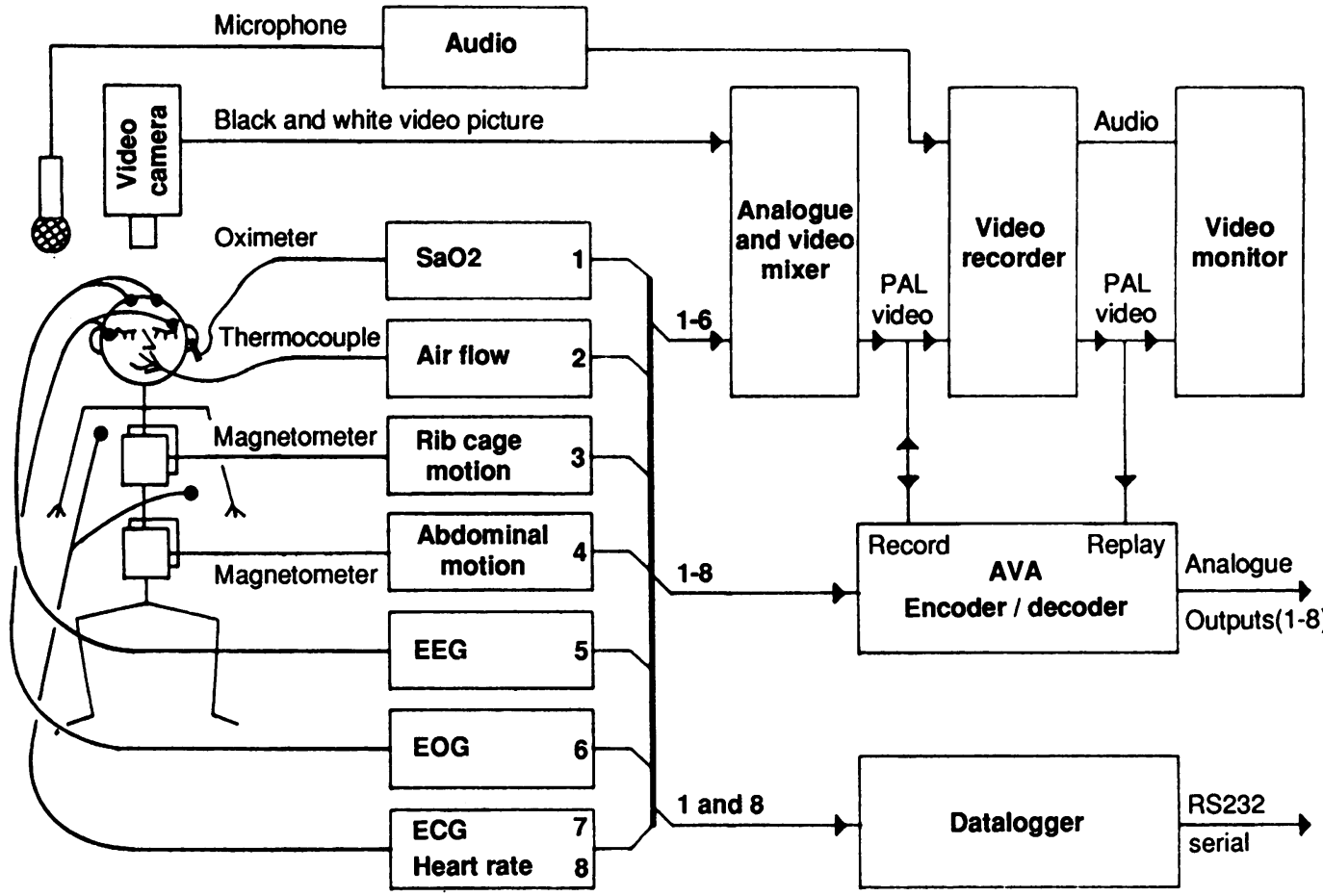

amplitude that can be recorded is \pm 1.5 volts, and noise on replay is less than $20 \mathrm{mv}$ (peak to peak).

The configuration of the system and the signals that we normally record are shown in figure $1 . \mathrm{SaO}_{2}$ is measured by ear or finger pulse oximeter, airflow at the nose and mouth by

thermocouples, and ribcage and abdominal anteroposterior motion by two pairs of magnetometers ${ }^{6}$; the electroencephalogram (EEG) (occipital notch-vertex), ${ }^{7}$ electro-oculogram (EOG), electrocardiogram (ECG), and heart rate are obtained by means of conventional electrodes and amplifiers. Alternative or

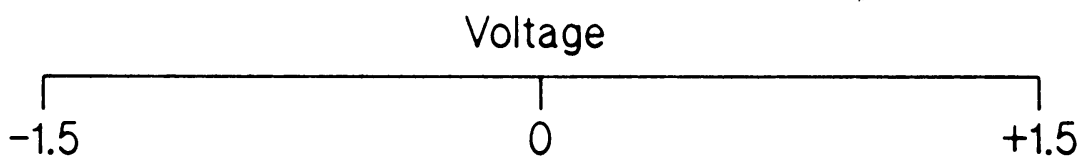

Ch1

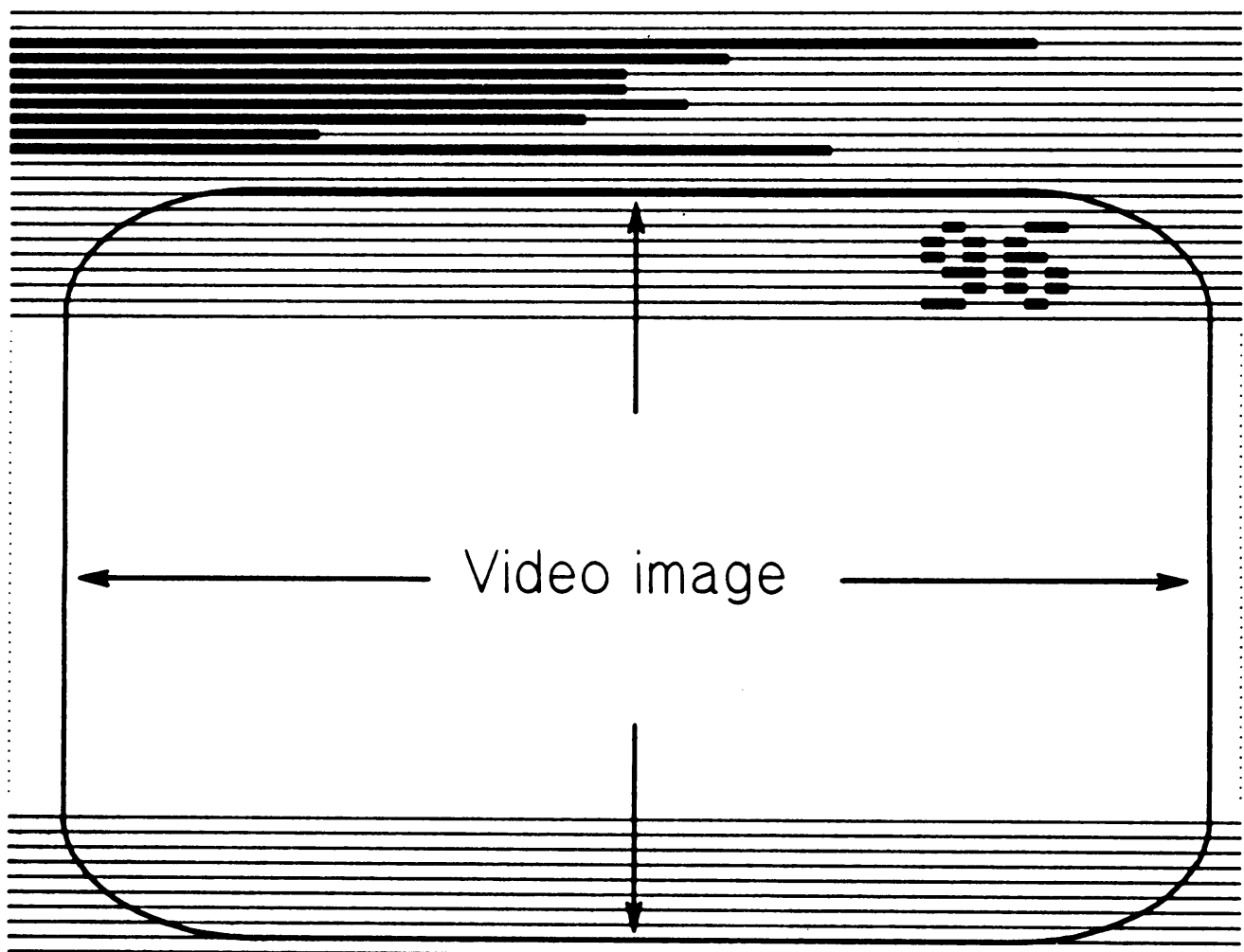


Figure 3 A single frame of the video recording of $a$ normal subject, with scrolling signals representing arterial oxygen saturation ( $\mathrm{SaO}_{2}$ : red), airflow (green), rib cage motion (cyan), abdominal motion (dark blue), electroencephalogram (magenta), and electro-oculogram (yellow). The horizontal distance between the graticule dots (see also fig 4) represents five seconds (total sweep $80 \mathrm{~s}$ ). The vertical distance between dots represents $25 \% \mathrm{SaO}_{2}$ $10 \%$ at the bottom and $100 \%$ at the top of the screen); $10 \mathrm{~mm}$ ribcage and abdominal movement; $200 \mu v E E G ; 1$ mv EOG, airflow is qualitative.

Digits represent

instantaneous $\mathrm{SaO}_{2}$ corresponding to real time at the right hand edge of the screen.

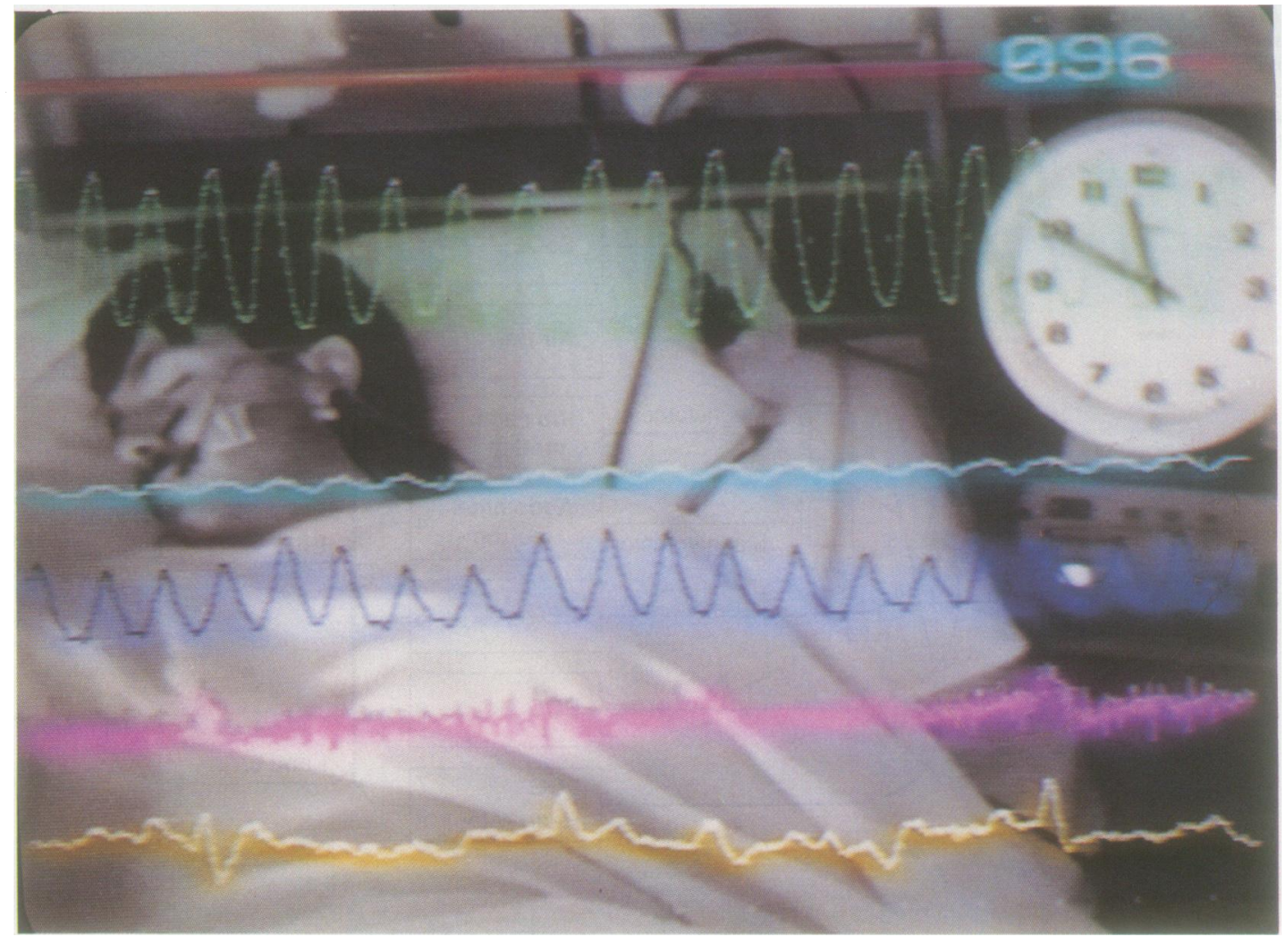

additional signals can be used: an inductance plethysmograph can be substituted for magnetometers and thermistors for thermocouples, and other EEG leads together with a submental or tibial electromyogram can be incorporated if required.

A low light video camera views the patient, with an infrared lamp providing illumination.
Time is monitored by the inclusion of a clock face in the image and warning lights related to the quality of the signal on the oximeter can be kept in view. An audio signal is obtained from a microphone positioned at the bedhead. The mixed video signal is displayed on a video monitor and, with the audio signal, is recorded on a normal VHS video recorder in long
Figure 4 A single frame of the video recording of $a$ subject with severe obstructive apnoea with calibration as in figure 3. Note that the subject has just taken three breaths after an episode of apnoea lasting over 60 seconds, during which arterial oxygen saturation $\left(\mathrm{SaO}_{2}\right)$ fell to $53 \%$; during the apnoea rib cage and abdominal motion continued out of phase (obstructive apnoea), and the electro-oculogram (EOG) is characteristic of rapid eye movement

(REM) sleep; the delay of about 10 seconds between resumption of airflow and the consequent increase in $\mathrm{SaO}_{2}$ is in part

instrumental and in part due to blood circulation time. The arrows indicate the five second period displayed in more detail in figure 5.

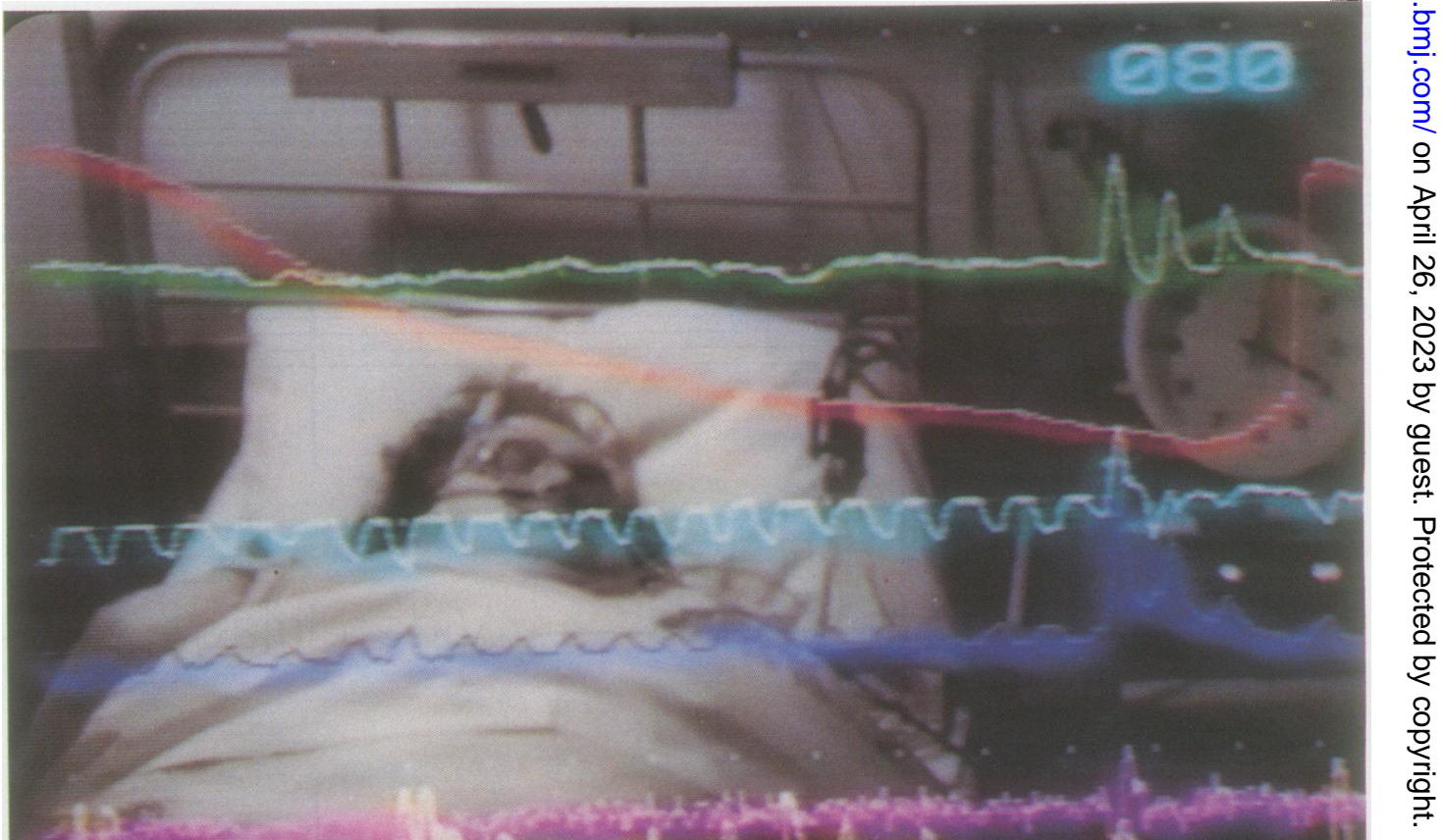




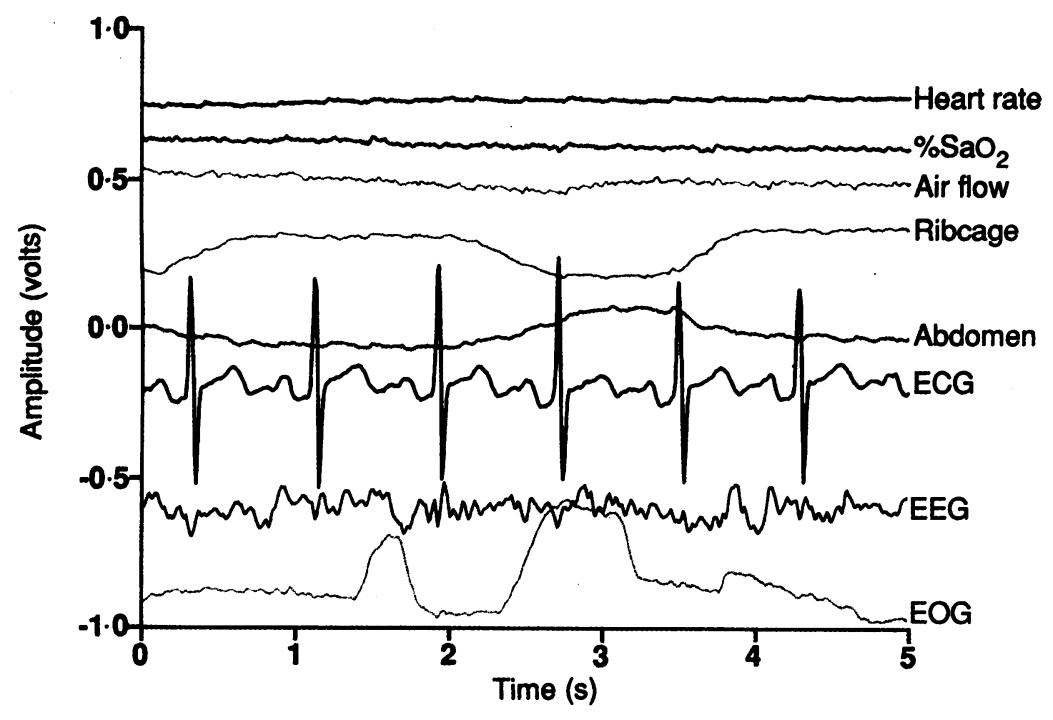

Figure 5 Example of signals replayed from the video tape by means of the analogue to visual to analogue encoder-decoder. The expanded time scale shows details of the faster changing waveforms; the five second period displayed corresponds to that indicated by the arrows on figure 4 . On the ordinate $0-1$ volt represents $0-100$ beats/min heart rate, $0-100 \%$ arterial oxygen saturation $\left(\mathrm{SaO}_{2}\right) ; 1$ volt peak to peak represents $1 \mathrm{~cm}$ of rib cage or abdominal movement, 1 mv electrocardiogram (ECG), $200 \mu v$

electroencephalogram (EEG), 1 mv electro-oculogram (EOG) (the baselines for these waveforms have been offset to make the figure clearer). Air flow is qualitative.

play mode. A standard E180 VHS video tape gives a recording of six hours and an E240 tape eight hours. Certain of the signals (usually $\mathrm{SaO}_{2}$ and heart rate) are recorded also by a data logger, the output of which gives a calibrated record plotted against time.

The recording devices, camera, and light source are assembled as a portable system on a trolley and studies are usually performed in a room with a single bed in the respiratory medicine ward. A video monitor is situated at the nurses' station so that the nursing staff can keep the subject under observation and replace sensors that come adrift.

Figure 3 shows a typical video frame from a recording of a normal subject and figure 4 a similar display from a patient with severe obstructive sleep apnoea. The quality of the waveforms reconstructed by means of the AVA encoder-decoder is illustrated in figure 5. This shows five seconds of recording from within the period of time illustrated in figure 4. The time axis has been magnified considerably to allow closer examination of the electrophysiological signals. Two additional signals not normally displayed as video images in our arrangement are included: the electrocardiogram and heart rate.

The signals recorded on video tape allow a full polysomnographic analysis to be performed. In our normal routine a technician analyses the video recording during normal working hours, initially by scanning the tape in "fast play" mode (10 times normal speed), using the record of $\mathrm{SaO}_{2}$ against time derived from the data logger as a guide. A note is kept of posture; number, type, and duration of apnoeic or hypopnoeic events; sleep stage; chest wall motion; unusual observations; and any other events of interest. The video display allows crude analysis of sleep stage (wakefulness and light, deep, and rapid eye movement sleep). More precise sleep staging or analysis of possible cardiac dysrhythmias may be obtained by displaying the reconstructed EEG and EOG or ECG signals on an oscilloscope. The $\mathrm{SaO}_{2}$ recording is analysed by computer to give a time weighted $\mathrm{SaO}_{2}$ profile, ${ }^{8}$ a mean value, and the percentage of sleep time during which $\mathrm{SaO}_{2}$ is less than $90 \%$. The report and clinical interpretation are done by hand.

Setting up and checking the equipment at the start of a study takes one to one and a half hours. Analysis time depends on the outcome: a normal result is analysed in less than one hour; for a patient with the severe sleep apnoea syndrome the full analysis requires two to two and a half hours.

\section{Discussion}

The use of video recording of patients with possible sleep apnoea is increasingly recommended, both for detailed polysomnography and in conjunction with oximetry as a simpler screening procedure. ${ }^{9}$ The system described here combines the simplicity of such screening methods with the more detailed information obtained by polysomnography and has the considerable advantage of allowing synchronisation and direct visualisation of the video image with the various analogue recordings. The audio signal provides useful information on snoring and associated sounds related to partial obstruction of the upper airway. During the night the visual display unit monitor allows supervision of the individual by the nursing staff and if on replay some signals are inadequate or lost the explanation is usually readily apparent. During analysis of the data direct observation of the patient helps in assessing the onset of sleep, the frequency of waking, and the degree of restlessness; and it shows the posture of the patient, which is an important determinant of the frequency and severity of episodes of apnoea. ${ }^{10}$

The addition of the AVA encoder-decoder to our original system ${ }^{5}$ allows more detailed analysis of signals with a faster time base where this is required (fig 5). The system is flexible in terms of the input recorded and retrieved. It is, for instance, readily adaptable for continuous monitoring of the pressure within the mask of a nasal continuous positive airway pressure system; for this purpose the small changes in mask pressure with inspiration and expiration can be substituted for thermocouples or thermistors to record airflow.

Some of the advantages and disadvantages of the system are as follows:

Advantages

1 The capital costs are comparatively low, with much of the system based on readily available electronic equipment. Running costs are low and data storage is much more economical than with paper records. The capital cost of the system is under $£ 5000$ for the video display mixer and the AVA encoder-decoder alone; a complete system, including oximeter, costs about $£ 12000$.

2 The system can be operated without specialised overnight supervision, in familiar ward surroundings. A monitor situated at the 
nurses' station allows ready recognition of signal failure.

3 The synchronisation of sound and vision with physiological signals greatly assists interpretation of events during sleep.

4 The equipment is mobile and the system is readily adapted to record various combinations of signals, depending on the clinical information required.

5 The system allows elegant demonstration of sleep apnoea and its treatment for educational purposes.

\section{Disadvantages}

1 In our normal routine we rely on nonexpert supervision overnight. In practice, however, misplacement of sensors is likely only with the oximeter probe and oronasal thermocouples; loss of such signals is readily recognisable and the sensor is easily replaced by nursing staff after minimal instruction.

2 The speed of "browsing through" the recordings is limited by video technology. (To some extent the hard copy of $\mathrm{SaO}_{2}$ and heart rate data compensates for this.) Future video storage techniques are likely to overcome this limitation in time. Analysis is manual rather than automated. (The availability of the analogue waveforms would, however, allow the development of automated analysis.)

The quality of the data obtained with the system has proved very acceptable in clinical practice. During 1986-9 124 video sleep studies were performed and only eight were classed as failures: in three the patient did not sleep long enough, in three displaced oximeters or flow sensors, or both, were not replaced, and in two the signals failed to be recorded on video tape for technical reasons (in one case failure of the mains supply switched off the recorder and in the other the "record inhibit" of a reused tape was inadequately disabled).

We would like to thank the technical staff of the medical physics and respiratory medicine departments and the nursing staff of ward 29, Freeman Hospital, without whose support the successful development and implementation of this system would not have been possible.

1 Farney RJ, Walker LE, Jensen RL, Walker JM. Ear oximetry to detect apnoea and differentiate rapid eye movement (REM) and non-REM (NREM) sleep. Chest 1986;89:533-9.

2 Cooper BG, Veale D, Griffiths CJ, Gibson GJ. Value of nocturnal oxygen saturation as a screening test for sleep apnoea [abstract]. Thorax 1990;45:337P.

3 Orr WC. Utilization of polysomnography in the assessment of sleep disorders. Med Clin N Am 1985;69:1153-67.

4 Griffiths CJ, Ramsden PD. Combined display of $x$-ray image and physiological waveforms: description of an "add on" unit for synchronous video-cystometry [abstract]. In: Proceedings of the International Continence [abstract]. In: Proceedings of
Society. London, 1985:375-6.

5 Griffiths CJ. Combined display of video image with superimposed analogue waveforms for clinical applications. Clin Phys Physiol Meas 1987;8:367-73.

6 Griffiiths CJ, Gilmartin JJ, Gibson GJ, Murray A. Measurement of chest wall movement: design, performance and clinical use of a four-channel magnetometer mance and clinical use of a four-channel magneto
instrument. Clin Phys Physiol Meas 1983;4:363-71.

7 Kiloh LG, McComas AJ, Osselton JW, Upton ARM. Clinical electroencephalography. 4th ed. London: Butterworths, 1981:77.

8 Slutsky AS, Strohl KP. Quantification of oxygen saturation during episodic hypoxemia. Am Rev Respir Dis 1980;121: 893-5.

9 Stradling JR. Disorders of ventilatory control and sleep apnoea syndrome. In: Brewis RAL, Gibson GJ, Geddes DM, eds. Respiratory medicine. London: Ballière Tindall, 1990:1352-72.

10 McEvoy RD, Sharp DJ, Thornton AT. The effects of posture on obstructive sleep apnoea. Am Rev Respir Dis 1986;133:662-6. 